\section{Total Synthesis of Pandamarine}

Key words

singlet oxygen

furan oxidation

spiroketalization

pandamarine

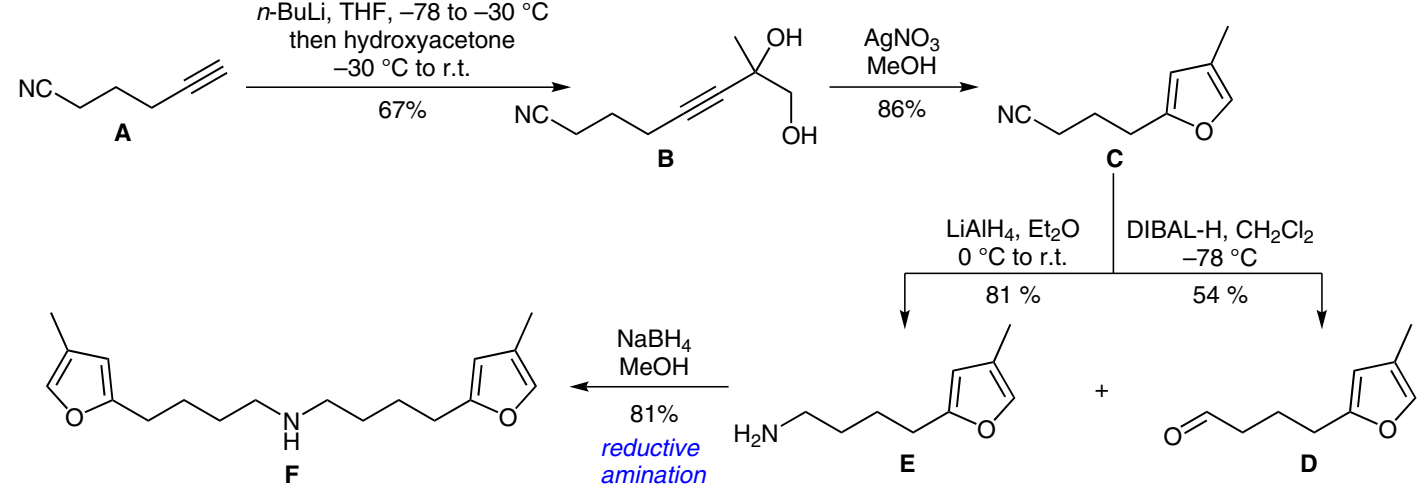

amination
Significance: The alkaloid pandamarine was isolated from leaves of the tropical plant Pandanus amaryllifolius, and features an interesting diazaspiro[4.5] core. After the group's report of the oxidation of furans to 5 -hydroxy-1H-pyrrol-2(5H)ones (Angew. Chem. Int. Ed. 2015, 54, 6283), they now disclose an elegant total synthesis of pandamarine relying on the double use of their methodology. Crucial to the success of the key transformation of bisfuran $\mathbf{F}$ to pandamarine is the dual role of methylene blue as both photosensitizer and redox catalyst.
Comment: Addition of excess alkyne A to hydroxyacetone furnished diol $\mathbf{B}$ which underwent silver-mediated cyclodehydration to furan C. Nitrile $\mathbf{C}$ was the precursor for both aldehyde $\mathbf{D}$ and amine $\mathbf{E}$, which were coupled by reductive amination to give bisfuran $\mathbf{F}$. In their key step, exposure of $\mathbf{F}$ to oxygen and methylene blue, followed by ammonia and dimethylsulfide gave intermediate bislactam $\mathbf{G}$. In situ methylene blue catalyzed radical oxidation with triplet oxygen and hydroperoxide reduction then furnished intermediate $\mathbf{I}$. Acidcatalyzed cyclization and elimination completed the synthesis of pandamarine in 30\% yield. 